OnLine Journal of Biological Sciences 10 (3): 114-125, 2010

ISSN 1608-4217

(C) 2010 Science Publications

\title{
Inhibition of U6 snRNA Transcription by PTEN
}

\author{
${ }^{1,3}$ Stephanie Cabarcas, ${ }^{2}$ Kounosuke Watabe and ${ }^{1}$ Laura Schramm \\ ${ }^{1}$ Department of Biological Sciences, St. John's University, Queens, New York 11439 \\ ${ }^{2}$ Department of Medical Microbiology and Immunology, \\ Southern Illinois University School of Medicine, Springfield, Illinois 62702 \\ ${ }^{3}$ National Cancer Institute, Laboratory of Cancer Prevention, Cancer Stem Cell Section, \\ 1050 Boyles Street, Building 560, Room 21-81, Frederick, MD 21702
}

\begin{abstract}
Problem statement: RNA polymerase III (RNA pol III) is responsible for transcribing many of the small structural RNA molecules involved in RNA processing and protein translation, thereby regulating the growth rate of a cell. RNA pol III transcribes both gene internal (tRNA) and gene external (U6 snRNA) promoters and proper initiation by RNA polymerase III requires the transcription initiation factor TFIIIB. TFIIIB has been shown to be a target of repression by tumor suppressors such as ARF, p53, RB and the RB-related pocket proteins. Also, TFIIIB activity is stimulated by the oncogenes c-Myc and the ERK mitogen-activated protein kinase. Recently, two TFIIIB subunits, BRF1 and BRF2, have been demonstrated to behave as oncogenes, making deregulation of TFIIIB activity and thus RNA pol III transcription an important step in tumor development. PTEN is a commonly mutated tumor suppressor regulating cell growth, proliferation and survival. Thus, we sought to examine the potential role of PTEN in regulating U6 snRNA transcription. Approach: We examined the potential for PTEN to regulate U6 snRNA transcription using in vitro RNA pol III luciferase assays, western blotting and deletion analysis in cancer cell lines differing in their PTEN status. Results: Using breast, cervical, prostate and glioblastoma cancer cells we demonstrate: (1) PTEN inhibition of gene external RNA pol III transcription is cell type specific, (2) PTEN-mediated inhibition of U6 transcription occurs via the C2 lipid-binding domain and (3) PTEN repression of U6 transcription occurs, at least in part, through the TFIIIB subunit BRF2. Conclusion/Recommendations: Our data demonstrates that regulation of the U6 snRNA gene by PTEN is mediated, in part by the TFIIIB oncogene BRF2, potentially identifying novel targets for chemotherapeutic drug design.
\end{abstract}

Key words: RNA polymerase, III transcription, PTEN, TFIIIB, BRF2

\section{INTRODUCTION}

RNA polymerase III (RNA pol III) transcribes genes involved in processing (U6 snRNA) and translation (tRNAs) and influences the growth rate of a cell (Schramm and Hernandez, 2002). RNA pol III recognizes different classes of promoters, which may be classified as gene internal, such as those found in the VAI and tRNA genes and gene external, as exemplified by the U6 snRNA gene (Schramm and Hernandez, 2002). TFIIIB is required for proper transcription initiation and in higher eukaryotes, at least two forms of TFIIIB have been identified (Schramm and Hernandez, 2002). Gene internal promoters, such as tRNA and VAI, require a form of TFIIIB consisting of TBP, Bdp1 and BRF1, whereas gene external promoters, such as
U6 snRNA, require a TFIIIB complex containing TBP, Bdp1 and BRF2 (Schramm and Hernandez, 2002).

RNA pol III activity is sensitive to growth conditions and is tightly regulated throughout the cell cycle; RNA pol III activity is lowest during mitosis, then increases slowly through G1 and reaches its maximal activity during the $\mathrm{S}$ and G2 phases (White et al., 1995; Leresche et al., 1996). As such, RNA pol III transcription is regulated by tumor suppressors and oncogenes, most often by modulating TFIIIB activity (White, 2005). TFIIIB is specifically targeted by the tumor suppressors ARF (Morton et al., 2007), p53 (Felton-Edkins et al., 2003a), RB (Felton-Edkins et al., 2003a) and the RB-related pocket proteins p107 and p130 (Sutcliffe et al., 1999) and more recently BRCA1 (Veras et al., 2009). TFIIIB activity is stimulated by the

Corresponding Author: Laura Schramm, Department of Biological Sciences, St. John's University, Queens, New York 11439 Tel: (718) 990-5558 Fax: (718) 990-5958 
oncogenes c-Myc (Felton-Edkins et al., 2003a), the casein kinase CK2 (Hu et al., 2004; Johnston et al., 2002) and the ERK (Felton-Edkins et al., 2003b) mitogen-activated protein kinase. RNA pol III transcription is also down-regulated by Maf1 during the growth cycle and in response to nutrient limitation, DNA damage, oxidative stress and a variety of drug treatments (Rollins et al., 2007; Johnson et al., 2007; Reina et al., 2006; Goodfellow et al., 2008). Maf1 represses RNA pol III transcription by targeting BRF2 (Rollins et al., 2007), BRF1 and RNA pol III directly (Reina et al., 2006). It has also been demonstrated that both gene internal (VAI) and gene external (U6 snRNA) RNA pol III transcription is regulated by chemopreventative agents such as EGCG (Jacob et al., 2007).

A definitive link between overexpression of RNA pol III product tRNAMet and transformation has been established (Marshall et al., 2008). Also, BRF2 expression and U6 snRNA gene external RNA pol III transcription correlate in all cancer cell lines tested implying a potential role for BRF2 as a regulator of cell proliferation (Cabarcas et al., 2008). More recently, BRF2 the TFIIIB subunit required for gene external RNA pol III transcription has been demonstrated to be over expressed in $40 \%$ of tumors from patients $(>330)$ with non-small cell lung cancer and induced expression of BRF2 in bronchial epithelial cells made normal cells behave like cancer cells (Lockwood et al., 2010). Taken together, these data suggest that TFIIIB deregulation may be an important step in cancer development. Thus, increasing our understanding of how TFIIIB is regulated and deregulated, by tumor suppressors such as PTEN may lead to novel chemotherapeutic drug development, ultimately leading to better patient outcomes.

The phosphatase and tensin homolog deleted on chromosome ten (PTEN) is a tumor suppressor regulating cell growth, proliferation and survival found in almost all tissues. Somatic mutations in PTEN frequently occur in breast, prostate, ovarian, endometrial and brain tumors (Leslie and Downes, 2004; Waite and Eng, 2002; Simpson and Parsons, 2001). Germline mutations in PTEN result in autosomal dominant disorders such as Bannayan-Zonana syndrome and Cowden syndrome (Waite and Eng, 2002; Simpson and Parsons, 2001). Cowden syndrome is a familial cancer predisposition syndrome which is characterized by the development of multiple hamartomas in the breast and thyroid (Waite and Eng, 2002; Simpson and Parsons, 2001). Bannayan-Zonana syndrome, an autosomal dominant hamartomous disease, is characterized by macrocephaly, lipomatosis and hemangiomatosis (Waite and Eng, 2002; Simpson and Parsons, 2001). Additionally, the use of murine model to study the effects of PTEN deletion and mutation further supports the role of PTEN as a tumor suppressor. For example, mouse studies demonstrate that PTEN deficiency can result in abnormal development of germinal layers, spontaneous development of both thyroid and colon tumors and thymic lymphomas (Waite and Eng, 2002). Long-term follow up of these mice demonstrated there was development of both breast and endometrial cancers which are characteristic of Cowden syndrome (Waite and Eng, 2002). Although murine models never exhibit the exact characteristics seen in either Cowden or Bannayan-Zonana syndrome, the development of abnormalities are reminiscent of both syndromes (Waite and Eng, 2002) and it is clear that PTEN deficiency results in both diseases.

The PTEN structure includes a lipid phosphatase domain, a C2-lipid binding domain and a C-terminal tail (Lee et al., 1999). As an antagonist of the Phosphatidyl Inositol 3-Kinase (PI3K)/Akt pathway, PTEN controls cell growth and its phosphatase domain is essential for tumor suppression (Dillon et al., 2007). The C2-lipid binding domain regulates cell migration (Raftopoulou et al., 2003) and has anti-oncogenic function (Okumura et al., 2005). The C-terminal tail PEST and PDZ domains regulate PTEN stability (Georgescu et al., 1999).

PTEN has been shown to repress gene internal RNA pol III transcription by targeting the TFIIIB subunits TBP, BRF1 and Bdp1 thereby, interfering with proper assembly of TFIIIB (Woiwode et al., 2008). Furthermore, Woiwode et al. (2008) demonstrated that the ability of PTEN to function as a repressor of endogenous precursor tRNALeu transcription is independent of p53 and can be uncoupled from PTENmediated effects on the cell cycle.

PTEN also inhibits RNA pol I transcription by decreasing promoter occupancy, through TBP (Zhang et al., 2005). As BRF2 is also a subunit of TFIIIB (Schramm and Hernandez, 2002), we speculated that gene external RNA pol III transcription may be regulated by PTEN, through TFIIIB. Using breast, cervical, prostate and glioblastoma cancer cells we sought to determine if PTEN regulates U6 snRNA transcription and if PTEN regulates gene external RNA pol III transcription whether it occurs via the BRF2 subunit. 


\section{MATERIALS AND METHODS}

Cell culture and luciferase assays: HeLa, DU-145, HCC1937, PC-3, MCF-7, MCF10A and U87 cells were obtained from the American Type Culture Collection (ATCC, Rockville, MD). All cell media was supplemented with 5\% FBS, L-glutamine, non-essential amino acids and penicillin/streptomycin (all supplies are from BioWhittaker, Walkersville, MD). The U6 and VAI luciferase vectors and transient transfections have been previously described (Rollins et al., 2007). Luciferase experiments were performed in triplicate, repeated three independent times and data presented are representative. Luciferase results are reported as Relative Light Units (RLU), representing the average of the Photinus pyralis firefly activity divided by the average of the Renilla luciferase vector. Statistical analysis was performed using one-way ANOVA with a Tukey post-test with a 95\% confidence interval (GraphpadPrism3.03, San Diego California, USA).

Mammalian expression constructs: pCDNA3PTEN was generated as previously described (Bandyopadhyay et al., 2004). Flag tagged PTEN and PTEN gross deletions were constructed using PCR primers designed with $\mathrm{Xba} 1$ and BamH1 restriction sites, subsequently cloned into Xba1 and BamH1 sites of the mammalian expression vector p3XFlag-CMV-7.1 (Sigma). FlagBRF2 was cloned as previously described (Rollins et al., 2007). pSG5L-HAPTEN C124S (Plasmid 10744); pSG5L-HAPTEN G129E (Plasmid 10746); pSG5LFlagHAPTEN G129R (Plasmid 10775), were all obtained from Addgene (Cambridge, MA).

Western blot analysis: Nuclear extracts were prepared from HCC1937 cells transfected with an empty Flag vector or Flag-tagged PTEN and western blot was performed using anti-actin and anti-flag antibodies as previously described (Rollins et al., 2007). Cytoplasmic and nuclear extracts were prepared from HeLa cells transfected with Flag-tagged PTEN or Flag-tagged C2PTEN and western blot was performed using antiflag antibodies as previously described (Rollins et al., 2007).

\section{RESULTS}

Inhibition of RNA pol III transcription by PTEN: To determine if PTEN can regulate gene external RNA pol III transcription, we monitored U6 snRNA transcription in a variety of cell lines differing in PTEN status. The HeLa cervical carcinoma cell line, DU-145 prostate carcinoma cell line, MCF-7 breast carcinoma cell line and MCF10A epithelial breast cell lines all express functional PTEN (Hlobilkova et al., 2000; Yu et al., 2002; McMenamin et al., 1999; Weng et al., 1999). We measured the effect of PTEN overexpression on both gene internal and external RNA pol III promoter activity in cells which express functional PTEN. HeLa, DU-145, MCF-7 and MCF10A cells were transfected with a promoterless pGL3 vector, pGL3-VAI (gene internal tRNA-like RNA pol III promoter) or pGL3-U6 (gene external RNA pol III promoter) and cotransfected with increasing concentrations of PTEN. Inhibition of VAI transcription by PTEN was statistically significant at the highest PTEN concentration tested in the HeLa cell line, $\mathrm{p}<0.05$ (Fig. 1A). In the DU145 cell line, VAI inhibition by PTEN was highly statistically significant at the two highest concentrations tested, $\mathrm{p}<0.001$ (Fig. 1A). In the MCF-7 cell line, VAI inhibition by PTEN was statistically significant, $p<0.01$ and in the MCF10A cell line, VAI inhibition by PTEN was statistically significant, at all concentrations tested, $\mathrm{p}<0.05$ and $\mathrm{p}<0.001$, respectively (Fig. 1A). Strikingly, PTEN mediated inhibition of U6 snRNA transcription was highly statistically significant, $\mathrm{p}<0.001$, in HeLa, DU-145, MCF-7 and MCF10A cells at almost all DNA concentrations tested (Fig. 1B). Interestingly, gene external RNA pol III transcription appears to be more sensitive to the effects of PTEN in HeLa, MCF-7 and MCF-10A cells.

Potentially the expression of both endogenous and exogenous PTEN together could influence the observed inhibition of both gene internal and external RNA pol III transcription. To rule out this possibility, we also measured the effect of PTEN overexpression on both gene internal and external RNA pol III promoter activity in the functionally null PTEN cells HCC1937, PC-3 and U-87. In the breast cancer HCC1937 cell line, VAI inhibition by PTEN was statistically significant, $p<0.01$, at the two highest concentrations tested (Fig. 2A). VAI inhibition by PTEN was statistically significant, $p<0.05$ and $\mathrm{p}<0.01$, at the two highest concentrations tested (Fig. 2A) in the prostate cancer cell line PC-3. Lastly, in the glioblastoma U87 cell line, VAI inhibition by PTEN was significant, $p<0.01$ and $p<0.001$ respectively (Fig. 2A). These data are in agreement with a recent report demonstrating inhibition of VAI transcription by PTEN (Woiwode et al., 2008). Interestingly, Fig. 1A and 2A suggests that the magnitude of PTEN repression of gene internal RNA pol III transcription may be tissue specific. 
OnLine J. Biol. Sci., 10 (3): 114-125, 2010

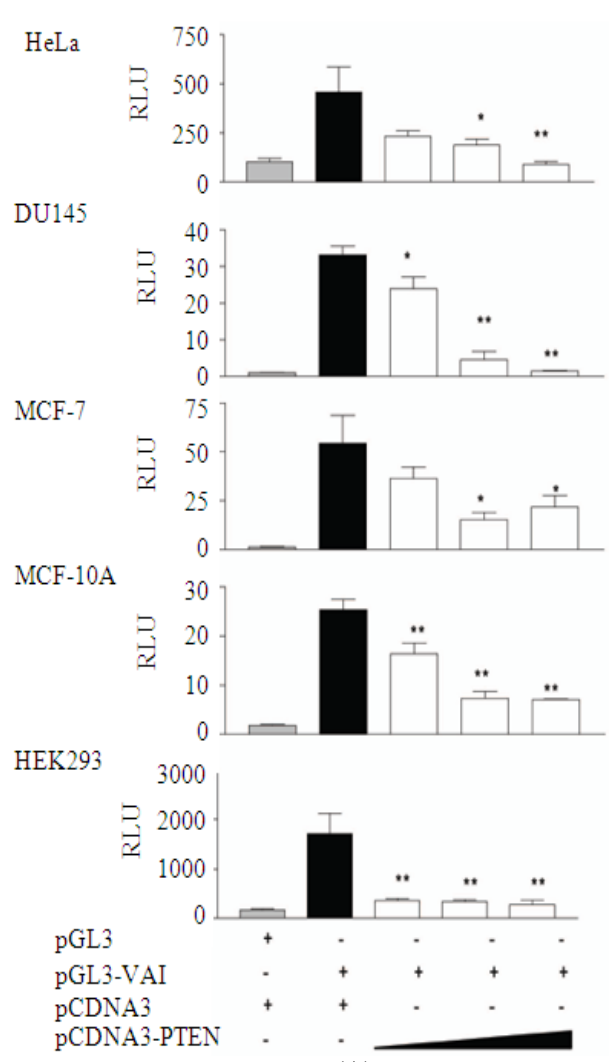

(A)
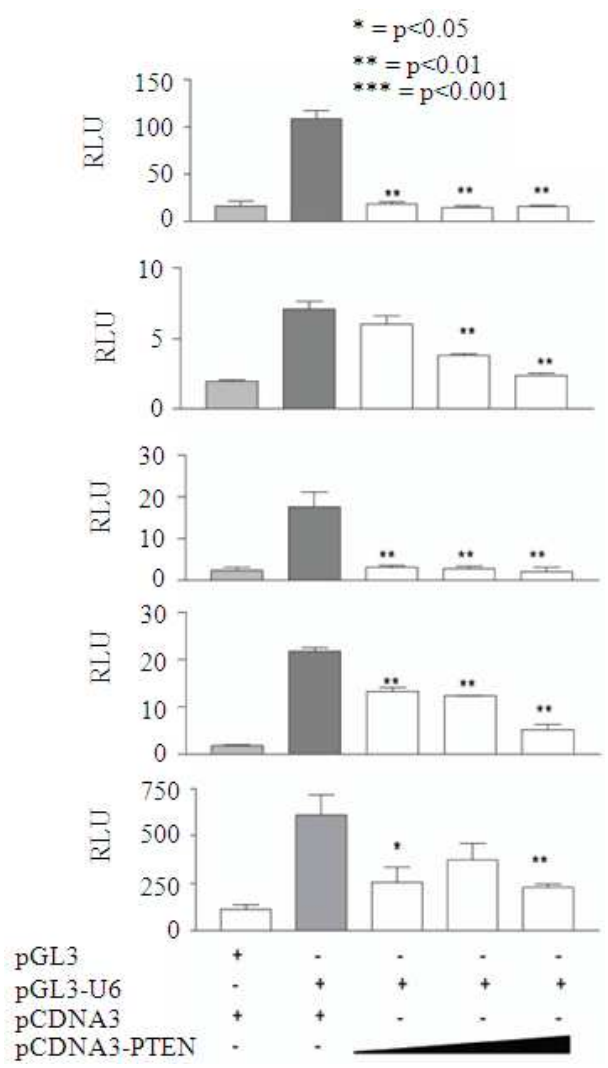

(B)

Fig. 1: PTEN inhibits RNA pol. III transcription in cancer cells which express functional PTEN. (A) HeLa, DU145, MCF-7 and MCF10A cells transiently transfected with empty pGL3 vector (100 ng), pGL3-VAI (100 ng) alone or co-transfected with increasing concentrations of pCDNA3-PTEN (100, 125, $150 \mathrm{ng})$; (B) HeLa, DU-145, MCF-7 and MCF10A cells transiently transfected with empty pGL3 vector (100 ng), pGL3-U6 $(100 \mathrm{ng})$ alone or cotransfected with increasing concentrations of pCDNA3-PTEN (50, 75, $100 \mathrm{ng})$. All luciferase assay results expressed as Relative Light Units (RLU): the average of the Photinus pyralis firefly activity observed divided by the average of the activity recorded from the Renilla luciferase vector. Experiments were done in triplicate, repeated three independent times and representative experiments are depicted. Statistical analysis was performed using oneway ANOVA with a Tukey post-test with a 95\% confidence interval (Graphpad Prism 3.03); ${ }^{*}=\mathrm{p}<0.05 ; * *=p<0.01 ; * * *=p<0.001$

Strikingly, PTEN mediated inhibition of U6 snRNA transcription in both PTEN null HCC1937 (breast) and PC-3 (prostate) cells was highly statistically significant (Fig. 2B). However, we did not see inhibition of U6 snRNA transcription by PTEN in PTEN null glioblastoma U87 cells (Fig. 2B). This data agrees with Woiwode et al. (2008) who did not see an effect on U6 transcription by PTEN in the U87 glioblastoma cell line. We cannot rule out the possibility that the effect of PTEN on U6 transcription may be cell type specific as we are able to show that PTEN significantly represses U6 transcription in a variety of cervical, prostate and breast carcinoma cell lines (Fig. 1B and 2B). Strikingly, for all breast and prostate cell lines tested, gene external RNA pol III transcription was more sensitive to inhibition by PTEN, as compared to gene internal RNA pol III transcription (Fig. 1 and 2).

To ensure that the observed repression of RNA pol III transcription was a result of exogenous PTEN expression, we prepared nuclear extracts from untransfected and transiently transfected HCC1937 cells with Flag-PTEN. Using an anti-flag antibody, we were able to detect PTEN in transiently transfected HCC1937 cells (Fig. 2C). The blots were probed with anti-actin as a loading control. Figure $2 \mathrm{C}$ confirms exogenous PTEN expression in the PTEN-null HCC1937 cells. 
OnLine J. Biol. Sci., 10 (3): 114-125, 2010
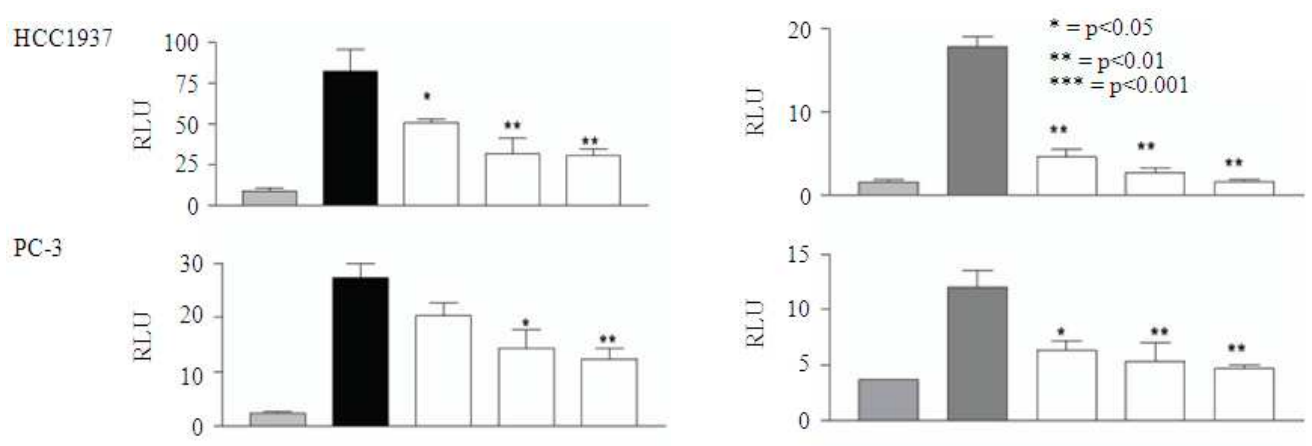

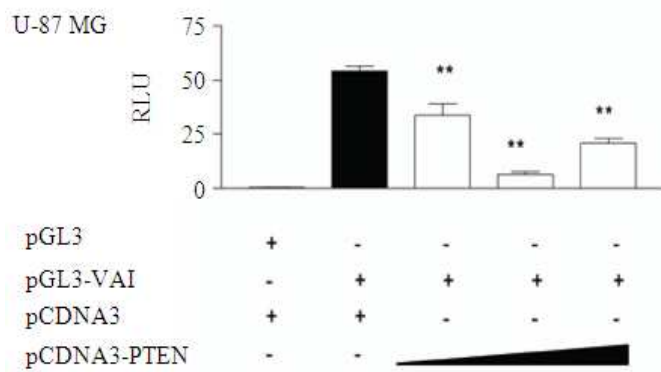

(A)

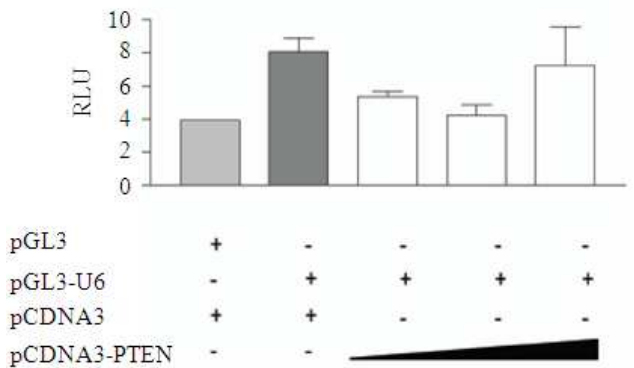

(B)

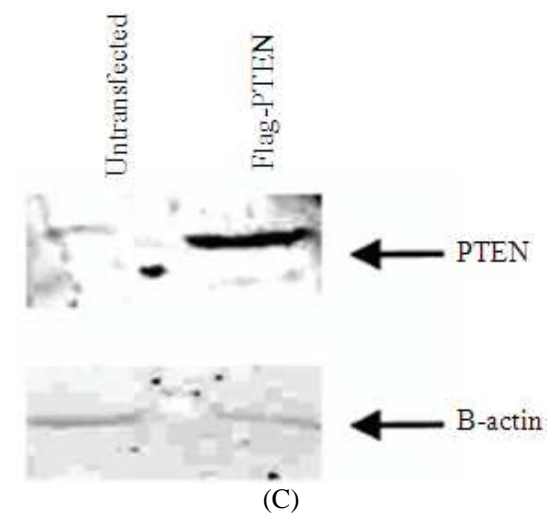

Fig. 2: PTEN inhibits RNA pol. III transcription in cancer cells which are PTEN-null. (A) PTEN-null HCC1937, PC-3 U87 cells transiently transfected with empty pGL3 vector (100 ng), pGL3-VAI (100 ng) alone or cotransfected with increasing concentrations of pCDNA3-PTEN (100, 125, $150 \mathrm{ng}$ ); (B) PTEN-null HCC1937, PC-3 and U87 cells transiently transfected with empty pGL3 vector (100ng), pGL3-U6 (100 ng) alone or co-transfected with increasing concentrations of pCDNA3-PTEN (50, 75,100 ng); (C) Nuclear extracts prepared from untransfected HCC1937 cells or HCC1937 cells transiently transfected with FlagPTEN and immunoblotted with anti-actin and anti-flag antibodies. All luciferase assay results expressed as Relative Light Units (RLU): the average of the Photinus pyralis firefly activity observed divided by the average of the activity recorded from the Renilla luciferase vector. Experiments were done in triplicate, repeated three independent times and representative experiments are depicted. Statistical analysis was performed using one-way ANOVA with a Tukey post-test with a 95\% confidence interval (Graphpad Prism $3.03) ; *=\mathrm{p}<0.05 ; * *=\mathrm{p}<0.01 ; * * *=\mathrm{p}<0.001$

To elucidate the mechanism (s) by which PTEN inhibits U6 snRNA transcription, we constructed gross PTEN deletions and tested their ability to inhibit gene internal and external RNA pol III transcription.
PTEN deletions inhibit U6 snRNA transcription: To determine the mechanism by which PTEN inhibits U6 transcription, we used previously characterized PTEN deletions (Odriozola et al., 2007), as depicted in Fig. 3A. 
OnLine J. Biol. Sci., 10 (3): 114-125, 2010

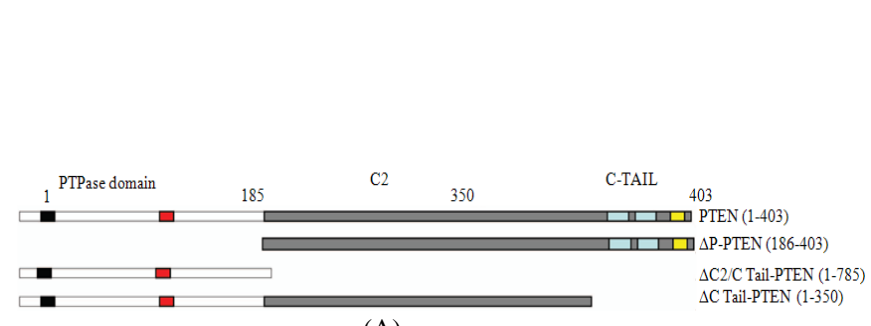

(A)

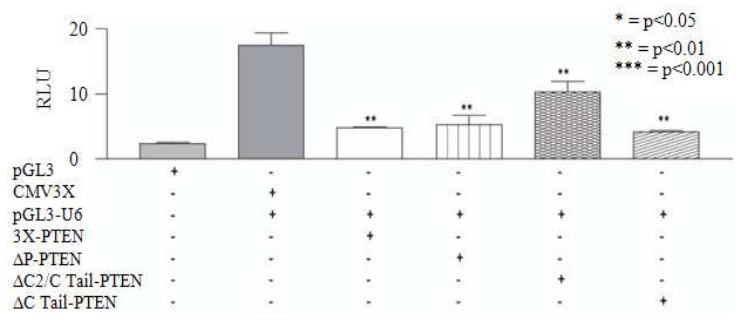

(B)

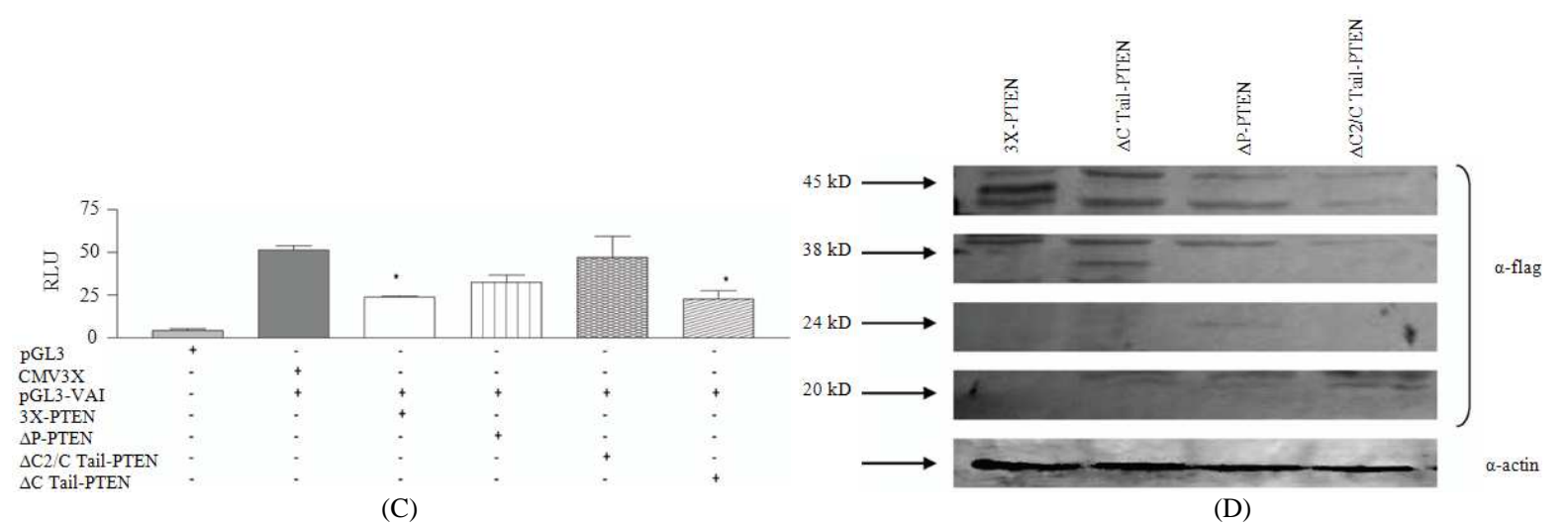

Fig. 3: U6 snRNA transcription is inhibited by PTEN deletions (A) Schematic representation of full-length PTEN and PTEN mutants. Full-length PTEN (1-403) represents the complete 403 amino acid protein. $\triangle$ P-PTEN (186-403) represents a deletion of the PTPase domain of PTEN. $\triangle$ C2/CTail-PTEN (1-185) represents a deletion of the $\mathrm{C} 2$ domain and the C-terminal tail. $\triangle \mathrm{CT}$ Tail-PTEN (1-351) represents a deletion of the Cterminal tail. White box corresponds to the phosphatase domain which contains a black box corresponding to the PIP2-binding domain and a red box corresponding to the catalytic domain. Grey box corresponds to the C2-terminal half of PTEN containing the C2-lipid binding domain; blue boxes correspond to the PEST sequences and the yellow box corresponds to the PDZ binding domain; (B) PTEN-null HCC1937 cells transiently transfected with empty pGL3 vector (100 ng), pGL3-U6 (100 ng) alone or co-transfected with 100 ng of the following: PTEN, $\triangle \mathrm{P}-\mathrm{PTEN}, \triangle \mathrm{C} 2 / \mathrm{CT}$ ail-PTEN and $\triangle \mathrm{CT}$ ail-PTEN; (C) PTEN-null HCC1937 cells transiently transfected with empty pGL3 vector (100 ng), pGL3-VAI (100 ng) alone or co-transfected with $150 \mathrm{ng}$ of the following: PTEN, $\triangle \mathrm{P}-\mathrm{PTEN}, \triangle \mathrm{C} 2 / \mathrm{CT}$ ail-PTEN and $\triangle \mathrm{CT}$ ail-PTEN. All luciferase assay results expressed as Relative Light Units (RLU): the average of the Photinus pyralis firefly activity observed divided by the average of the activity recorded from the Renilla luciferase vector. Experiments were done in triplicate, repeated three independent times and representative experiments are depicted. Statistical analysis was performed using one-way ANOVA with a Tukey post-test with a $95 \%$ confidence interval (Graphpad Prism 3.03); * $=\mathrm{p}<0.05 ; * *=\mathrm{p}<0.01 ; * * *=\mathrm{p}<0.001$

The PTEN deletions include: $\triangle \mathrm{P}$-PTEN, $\triangle \mathrm{C} 2 / \mathrm{CT}$ TailPTEN and $\triangle$ CTail-PTEN. $\triangle \mathrm{P}$-PTEN lacks the phosphatase domain and has been previously characterized for PTEN interaction (Odriozola et al., 2007) and membrane binding studies (Das et al., 2003). $\triangle \mathrm{C} 2 / \mathrm{CT}$ ail-PTEN lacks the C2-lipid binding domain essential for proper positioning at the cell membrane and regulating cell migration (Raftopoulou et al., 2003) as well as the C-terminal tail containing PEST and PDZ domains responsible for PTEN stability (Georgescu et al., 1999). $\triangle$ CTail-PTEN lacks the C- terminal tail and has previously been used in cell membrane binding studies (Das et al., 2003).

HCC1937 cells were transiently transfected with pGL3-U6 or pGL3-VAI and with full-length PTEN or PTEN deletions. U6 snRNA transcription is highly statistically inhibited by the PTEN mutants $\triangle$ P-PTEN and $\triangle$ CTail-PTEN, p<0.001, Fig. 3B. $\triangle$ P-PTEN lacks the phosphatase domain and significantly inhibits U6 snRNA transcription, $\mathrm{p}<0.001$, suggesting that the phosphatase domain may not be the primary PTEN domain responsible for gene external RNA pol III 
inhibition. $\triangle$ CTail-PTEN, which has an intact $\mathrm{C} 2$ domain but lacks the C-terminal tail, also severely debilitates U6 snRNA transcription. Interestingly, U6 snRNA transcription is only slightly inhibited by PTEN deletion $\triangle \mathrm{C} 2 / \mathrm{CT}$ ail-PTEN, which expresses the phosphatase domain alone $\mathrm{p}<0.05$, Fig. 3B. Strikingly, U6 snRNA transcription is more sensitive to repression by PTEN mutants expressing the C2 domain, suggesting that the $\mathrm{C} 2$ domain may play a pivotal role in repressing gene external RNA pol III transcription.

To examine which portion of PTEN may be responsible for VAI inhibition, HCC1937 cells were transiently transfected with pGL3-VAI and with full length PTEN or PTEN deletions. VAI transcription is significantly inhibited by full-length PTEN and $\triangle \mathrm{CT}$ TailPTEN, p<0.05 (Fig. 3C). The PTEN mutant $\triangle \mathrm{P}$-PTEN, lacking the phosphatase domain, is incapable of inhibiting VAI transcription (Fig. 3C) suggesting a role for the phosphatase domain in VAI mediated inhibition. This data is in agreement with a recent report demonstrating that the phosphatase domain is responsible for VAI inhibition (Woiwode et al., 2008). The $\Delta \mathrm{C} 2 / \mathrm{CT}$ ail-PTEN mutant, lacking the $\mathrm{C} 2$ and $\mathrm{C}$ tail domains, failed to inhibit VAI transcription. Thus, the possibility exists that the phosphatase domain itself may be unable to inhibit VAI transcription but other PTEN functional domains may be required to inhibit gene external RNA pol III transcription. Combined with the observation that deletion of the phosphatase domain did not affect PTEN's ability to inhibit U6 transcription (Fig. 3B) we further investigated the potential role of the PTEN phosphatase domain in regulating RNA pol III transcription, by testing several well characterized point mutations in the lipid phosphatase domain (Bandyopadhyay et al., 2004).

HCC1937 cells were transiently transfected with pGL3-U6 and with the well-characterized PTEN phosphatase domain mutants: G129E, G129R and C124S (Fig. 4A) (Ramaswamy et al., 1999). The PTEN phosphatase point mutations used in this study are depicted in Fig. 4. G129R is defective for both lipid binding and protein phosphatase activities. G129E, defective for lipid phosphatase activity, was previously used to demonstrate that PTEN mediated repression of gene internal RNA pol III transcription requires lipid phosphatase activities (Woiwode et al., 2008; Zhang et al., 2005). C124S, a phosphatase deficient and catalytically inactive mutant was tested as well (Ramaswamy et al., 1999). Figure 4A demonstrates that neither the lipid nor protein phosphatase activities are required for U6 inhibition by PTEN as G129E, G129R and C124S all significantly inhibit U6 activity, $\mathrm{p}<0.001$, Fig. 4A. These data suggest that the PTEN phosphatase domain is not responsible for the observed gene external RNA pol III transcription inhibition by PTEN.

In the case of VAI transcription, Fig. 3B suggested that the phosphatase domain may be responsible for the observed inhibition of gene internal RNA pol III transcription. To further examine the role of the PTEN phosphatase domain in regulating gene internal RNA pol III transcription, we transiently transfected HCC1937 cells with pGL3-VAI and the well characterized phosphatase domain mutants, G129E, G129R and C124S, Fig. 4B. Figure 4B demonstrates that none of the phosphatase point mutations tested are capable of inhibiting VAI activity. These data suggest that mutation or complete deletion of the phosphatase domain compromises the ability of PTEN to inhibit gene internal RNA pol III transcription, Fig. 3B and 4B. This data is in agreement with the data presented by Woiwode et al. (2008) who demonstrated a role for the phosphatase domain of PTEN in inhibiting gene internal RNA pol III transcription.

These data suggest that the phosphatase activities of PTEN are responsible for VAI inhibition but do not play a role in inhibition of gene external U6 snRNA transcription, Fig. 3-4. Interestingly, in the case of U6 snRNA transcription, the $\triangle \mathrm{C} 2 / \mathrm{CT}$ ail-PTEN mutant inhibits, $\mathrm{p}<0.05$, much less than full-length PTEN, $\triangle \mathrm{P}$ PTEN and $\triangle$ CTail-PTEN $(\mathrm{p}<0.001)$ (Fig. 3A). This suggests the $\mathrm{C} 2$ domain of PTEN may be important in regulating gene external RNA pol III transcription. To test this hypothesis, we generated a previously characterized mutant expressing the PTEN C2 domain alone (Chu et al., 1997) and tested its ability to affect RNA pol III transcription.

The C2 domain is responsible for PTEN inhibition of U6 snRNA transcription: The C2 domain can regulate cell migration (Raftopoulou et al., 2003) and has anti-oncogenic function independent of the phosphatase activity (Okumura et al., 2005). To determine if the $\mathrm{C} 2$ domain alone could inhibit U6 snRNA transcription we constructed a previously characterized C2 domain construct (Fig. 5) (Raftopoulou et al., 2003; Das et al., 2003). HCC1937 cells were transiently transfected with pGL3-U6 and full-length PTEN or pGL3-U6 and C2-PTEN. Strikingly, the C2 domain alone is capable of significantly inhibiting U6 promoter activity, $\mathrm{p}<0.001$ (Fig. 5A). 
OnLine J. Biol. Sci., 10 (3): 114-125, 2010
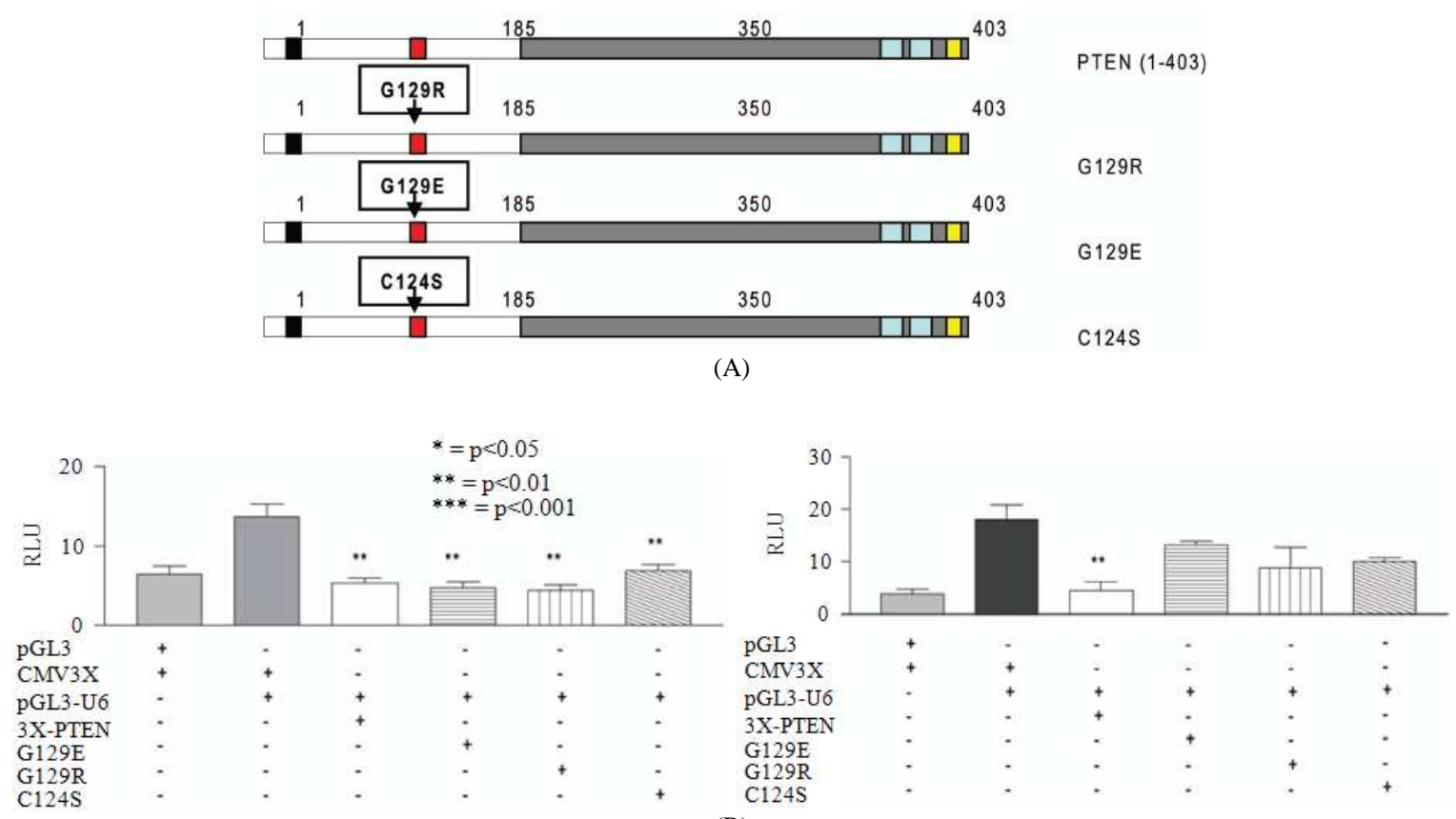

(B)

Fig. 4: PTEN phosphatase point mutations inhibit U6 snRNA transcription. Schematic of well-characterized PTEN phosphatase point mutations in the catalytic site. (A) PTEN-null HCC1937 cells transiently transfected with empty pGL3 vector (100 ng), pGL3-U6 (100 ng) alone or co-transfected with $100 \mathrm{ng}$ of the following: following: pSG5L HA PTEN G129E; pSG5L Flag HA PTEN G129R; pSG5L HA PTEN C124S; (B) PTENnull HCC1937 cells transiently transfected with empty pGL3 vector (100 ng), pGL3-VAI (100 ng) alone or co-transfected with $150 \mathrm{ng}$ of the following: following: pSG5L HA PTEN G129E; pSG5L Flag HA PTEN G129R; pSG5L HA PTEN C124S. All luciferase assay results expressed as Relative Light Units (RLU): the average of the Photinus pyralis firefly activity observed divided by the average of the activity recorded from the Renilla luciferase vector. Experiments were done in triplicate, repeated three independent times and representative experiments are depicted. Statistical analysis was performed using one-way ANOVA with a Tukey post-test with a 95\% confidence interval (Graphpad Prism 3.03); * $=\mathrm{p}<0.05 ; * *=\mathrm{p}<0.01$; $* * *=\mathrm{p}<0.001$

We further tested if the $\mathrm{C} 2$ domain plays a role in PTEN mediated inhibition of VAI activity, we transiently transfected HCC1937 cells with pGL3-VAI and full-length PTEN or pGL3-VAI and C2-PTEN. Figure 5B demonstrates the C2-PTEN construct is not capable of inhibiting VAI activity, further confirming the role of the PTEN phosphatase domain in regulating gene internal RNA pol III transcription. The observation that the C2-PTEN mutant preferentially inhibits U6 snRNA transcription, but not VAI, suggests that the mutant is indeed being expressed (Fig. 5A-B). However, to confirm the expression and localization of the C2-PTEN mutant, nuclear and cytoplasmic extracts from HeLa cells transiently transfected with Flag alone or FlagC2PTEN were prepared and western blotting confirmed that the $\mathrm{C} 2$ mutant localized to the cell nucleus (Fig. 5C).
How PTEN enters the nucleus appears to be influenced by a variety of mechanisms (Planchon et al., 2008). NLS-like sequences within PTEN and amino acids 265-269, which are within the $\mathrm{C} 2$ domain, were shown to be necessary for nuclear import (Chung et al., 2005). Also, the $C 2$ domain has been shown to interact with the Major Vault Protein (MVP) in HeLa cells providing a mechanism by which PTEN can enter the nucleus (Yu et al., 2002). MVP is one of three proteins which comprise mammalian vaults, a ribonucleoprotein particle which is proposed to function as a nuclearcytoplasmic transporter (Mossink et al., 2003). Thus, we speculate that the $\mathrm{C} 2$ domain of PTEN may be capable of repressing RNA pol III transcription by entering the nucleus via the MVP or the NLS-like sequence within amino acids 265-269. 


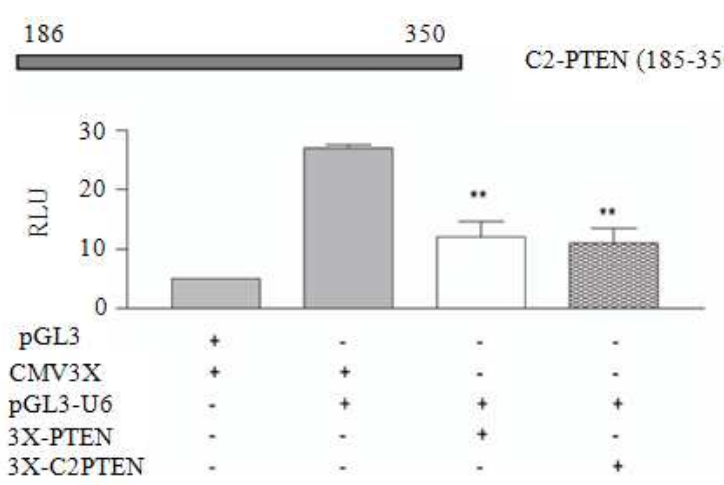

(A)
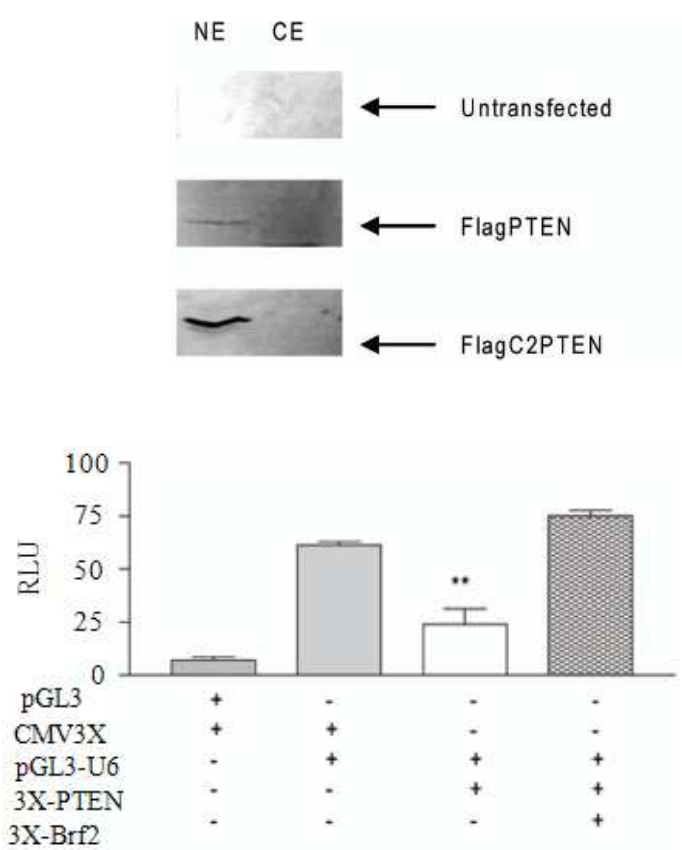

(D)

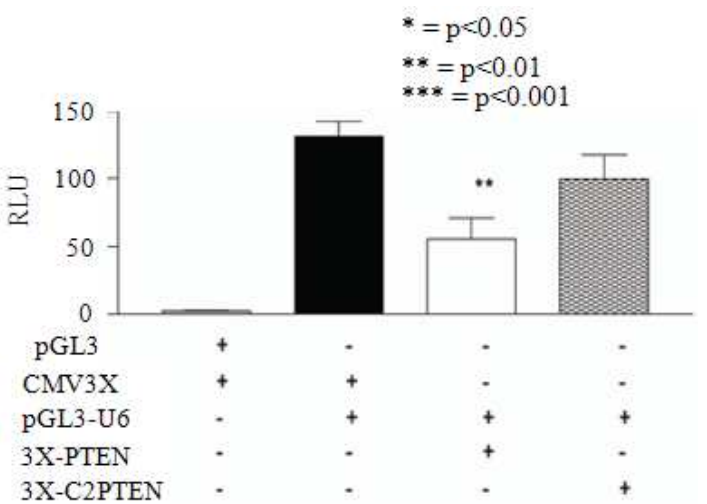

(B)

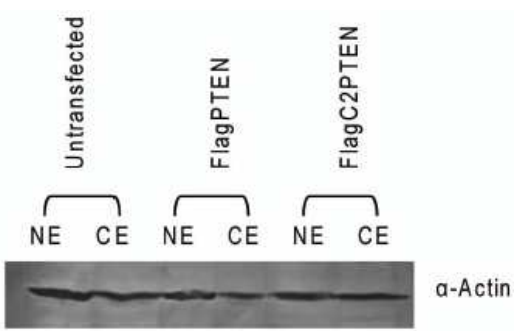

(C)

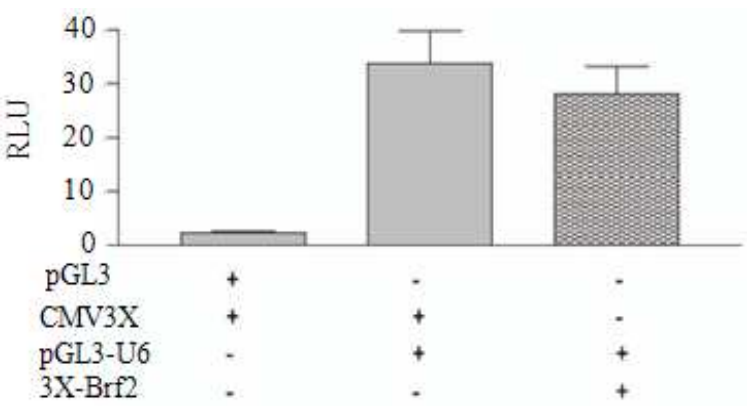

(E)

Fig. 5: The C2 domain is responsible for PTEN inhibition of U6 snRNA transcription (A) Schematic of C2-PTEN domain alone. PTEN-null HCC1937 cells transiently transfected with empty pGL3 vector (100 ng), pGL3U6 (100 ng) alone or co-transfected with $100 \mathrm{ng}$ of full-length PTEN or C2-PTEN; (B) PTEN-null HCC1937 cells transiently transfected with empty pGL3 vector (100 ng), pGL3-VAI (100 ng) alone or co-transfected with $150 \mathrm{ng}$ of full-length PTEN or C2-PTEN; (C) Nuclear and cytoplasmic extracts prepared from untransfected HeLa cells or HeLa cells transiently transfected with Flag-PTEN and Flag-C2PTEN and immunoblotted with anti-actin and ntiflag antibodies; (D) PTEN-null HCC1937 cells transiently transfected with pGL3 (100 ng), pGL3-U6 (100 ng) alone, pGL3-U6 (100 ng) and full-length PTEN, or co-transfected with pGL3-U6, full-length PTEN and BRF2; (E) PTENnull HCC1937 cells transiently transfected with pGL3 (100 ng), pGL3-U6 (100ng) alone, or co-transfected with pGL3-U6 and BRF2. All luciferase assay results expressed as Relative Light Units (RLU): the average of the 10 Photinus pyralis firefly activity observed divided by the average of the activity recorded from the Renilla luciferase vector. Experiments were done in triplicate, repeated three independent times and representative experiments are depicted. Statistical analysis was performed using one-way ANOVA with a Tukey post-test with a $95 \%$ confidence interval (Graphpad Prism 3.03); $*=\mathrm{p}<0.05 ; * *=\mathrm{p}<0.01 ; * * *=\mathrm{p}<0.001$ 
The data presented suggests a role for the $\mathrm{C} 2$ domain in PTEN-mediated regulation of gene external RNA pol III transcription. We demonstrate that the C2 domain itself is capable of regulating U6 snRNA transcription (Fig. 5A). The precise mechanism by which PTEN inhibits gene external RNA pol III transcription has yet to be elucidated, but the observed inhibition of U6 and VAI transcription by PTEN was not equivalent (Fig. 1 and 2), suggesting that the RNA pol III enzyme itself may not be the primary target. Thus, we speculate that regulation of gene external RNA pol III transcription by PTEN may involve TFIIIB.

PTEN repression of U6 snRNA transcription occurs via the TFIIIB subunit BRF2: To determine if PTEN inhibits U6 snRNA transcription through TFIIIB, we overexpressed the TFIIIB subunit BRF2 in HCC1937 cells. HCC1937 cells were transiently transfected with pGL3-U6, pGL3-U6 and PTEN, or pGL3-U6, PTEN and BRF2. Interestingly, overexpression of the TFIIIB subunit BRF2 (Fig. 5D) is capable of alleviating PTEN mediated repression of U6 snRNA transcription and restoring transcription to levels of pGL3-U6 alone. To ensure that BRF2 was not limiting in our assay, we transiently transfected HCC1937 cells with pGL3-U6 alone and co-transfected with pGL3-U6 and BRF2 (Fig. 5E). As demonstrated, co-transfecting BRF2 with pGL3-U6 does not increase activity of U6 (Fig. 5E). These data suggest that PTEN exerts its repressional activity, at least in part, through the TFIIIB subunit BRF2, recently demonstrated to be an oncogene (Lockwood et al., 2010).

\section{DISCUSSION}

Here we report the inhibition of U6 snRNA transcription by PTEN in cervical, prostate and breast cancer cell lines. We also show that in breast and prostate cancer cells U6 snRNA transcription is more sensitive to PTEN mediated inhibition than VAI transcription (Fig. 1 and 2). Using previously characterized PTEN domain deletions and phosphatase point mutations, we demonstrate that the phosphatase domain is responsible for repression of VAI promoter activity (Fig. 3B and 4B) and further demonstrate that the $\mathrm{C} 2$ domain can independently repress $\mathrm{U} 6$ promoter activity (Fig. 3A and 5A). PTEN has been demonstrated to inhibit cell migration through its $\mathrm{C} 2$ domain, independent of its lipid phosphatase activity and that the control of cell migration through its $\mathrm{C} 2$ domain is expected to be an important feature of its tumor suppressor activity (Raftopoulou et al., 2003; Freeman et al., 2003). Additionally, Woiwode et al.
(2008) demonstrated that the ability of PTEN to inhibit tRNALeu transcription is independent of p53, we speculate that PTEN-mediated inhibition of U6 snRNA transcription in HCC1937 cells may also be independent of p53 as HCC1937 cells are p53 null (DelloRusso et al., 2007).

This report demonstrates a novel role for the $\mathrm{C} 2$ domain of PTEN, independent of its phosphatase activity, as a regulator of U6 transcription. We further demonstrate that overexpression of the TFIIIB subunit BRF2 can alleviate PTEN inhibition of U6 snRNA transcription (Fig. 5D). As both gene internal and external TFIIIB complexes share TBP and Bdp1, it is possible PTEN may regulate U6 snRNA transcription via TBP and Bdp1 as well and remains to be tested.

We speculate that the observed PTEN tissue specific repression of gene internal and gene external RNA pol III transcription occurs via two independent mechanisms. In the case of VAI transcription, the lipid phosphatase domain of PTEN is required (Fig. 3D and 4C) and inhibition occurs via the TFIIIB subunits BRF1 and Bdp1 (Woiwode et al., 2008). Inhibition of U6 snRNA transcription requires the $\mathrm{C} 2$ domain of PTEN (Fig. 3B and 5A) and occurs via the TFIIIB subunit BRF2 (Fig. 5D).

\section{CONCLUSION}

This report, to the best of our knowledge, provides the first evidence demonstrating tissue specific regulation of U6 snRNA transcription by PTEN, via the TFIIIB oncogene BRF2. We also demonstrate a novel role for the $\mathrm{C} 2$ domain of PTEN in the regulation of U6 snRNA transcription. Together, these data provide evidence for the rationale design of novel cancer therapeutics.

\section{ACKNOWLEDGMENT}

This study was supported in part by the Henry Luce foundation (LS and SC), a St. John's University faculty seed grant (LS) and NIH 1R15CA133842-01A1 (LS). Authors declare no conflict of interest.

\section{REFERENCES}

Bandyopadhyay, S., S.K. Pai, S. Hirota, S. Hosobe and T. Tsukada et al., 2004. PTEN up-regulates the tumor metastasis suppressor gene Drg-1 in prostate and breast cancer. Cancer Res., 64: 7655-7660. DOI: 10.1158/0008-5472.CAN-04-1623

Cabarcas, S., J. Jacob, I. Veras and L. Schramm, 2008. Differential expression of the TFIIIB subunits Brf1 and Brf2 in cancer cells. BMC Mol. Biol., 9: 74-74. DOI: $10.1186 / 1471-2199-9-74$ 
Chu, W.M., Z. Wang, R.B. Roeder and C.W. Schmid, 1997. RNA polymerase III transcription repressed by $\mathrm{Rb}$ through its interactions with TFIIIB and TFIIIC2. J. Biol. Chem., 272: 14755-14761. DOI: 10.1074/jbc.272.23.14755

Chung, J.H., M.E. Ginn-Pease and C. Eng, 2005. Phosphatase and tensin homologue deleted on chromosome 10 (PTEN) has nuclear localization signal-like sequences for nuclear import mediated by major vault protein. Cancer Res., 65: 4108-4116. DOI: 10.1158/0008-5472.CAN-05-0124

Das, S., J.E. Dixon and W. Cho, 2003. Membranebinding and activation mechanism of PTEN. PNAS, 100: 7491-7496. DOI: 10.1073/pnas.0932835100

DelloRusso, C., P.L. Welcsh, W. Wang, R.L. Garcia and M.C. King et al., 2007. Functional characterization of a novel BRCA1-null ovarian cancer cell line in response to ionizing radiation. Mol. Cancer Res., 5: 35-45. PMID: 17259345

Dillon, R.L., D.E. White and W.J. Muller, 2007. The phosphatidyl inositol 3-kinase signaling network: Implications for human breast cancer. Oncogene, 26: 1338-1345. DOI: 10.1038/sj.onc. 1210202

Felton-Edkins, Z.A., N.S. Kenneth, T.R. Brown, N.L. Daly and N. Gomez-Roman et al., 2003a. Direct regulation of RNA polymerase III transcription by RB, p53 and c-Myc. Cell Cycle, 2: 181-184. PMID: 12734418

Felton-Edkins, Z.A., J.A. Fairley, E.L. Graham, I.M. Johnston and R.J. White et al., 2003b. The Mitogen-Activated Protein (MAP) kinase ERK induces tRNA synthesis by phosphorylating TFIIIB. EMBO J., 22: 2422-2432. DOI: 10.1093/emboj/cdg240

Freeman, D.J., A.G. Li, G. Wei, H.H. Li and N. Kertesz et al., 2003. PTEN tumor suppressor regulates p53 protein levels and activity through phosphatasedependent and-independent mechanisms. Cancer Cell,3:117-130.DOI: 10.1016/S1535-6108(03)00021-7

Georgescu, M.M., K.H. Kirsch, T. Akagi, T. Shishido and H. Hanafusa, 1999. The tumor-suppressor activity of PTEN is regulated by its carboxylterminal region. Proc. Natl. Acad. Sci. USA., 96: 10182-10187. PMID: 10468583

Goodfellow, S.J., E.L. Graham, T. Kantidakis, L. Marshall and B.A. Coppins et al., 2008. Regulation of RNA polymerase III transcription by maf1 in mammalian cells. J. Mol. Biol., 378: 481-491. DOI: 10.1016/j.jmb.2008.02.060

Hlobilkova, A., P. Guldberg, M. Thullberg, J. Zeuthen and J. Lukas et al., 2000. Cell cycle arrest by the PTEN tumor suppressor is target cell specific and may require protein phosphatase activity. Exp. Cell Res., 256: 571-577. DOI: 10.1006/excr.2000.4867
Hu, P., K. Samudre, S. Wu, Y. Sun and N. Hernandez, 2004. CK2 phosphorylation of Bdp1 executes cell cycle-specific RNA polymerase III transcription repression. Mol. Cell, 16: 81-92. DOI: 10.1016/j.molcel.2004.09.008

Jacob, J., S. Cabarcas, I. Veras, N. Zaveri and L. Schramm, 2007. The green tea component EGCG inhibits RNA polymerase III transcription. Biochem. Biophys. Res. Commun., 360: 778-783. DOI: 10.1016/j.bbrc.2007.06.114

Johnson, S.S., C. Zhang, J. Fromm, I.M. Willis and D.L. Johnson, 2007. Mammalian Maf1 is a negative regulator of transcription by all three nuclear RNA polymerases. Mol. Cell, 26: 367-379. DOI: 10.1016/j.molcel.2007.03.021

Johnston, I.M., S.J. Allison, J.P. Morton, L. Schramm and P.H. Scott et al., 2002. CK2 forms a stable complex with TFIIIB and activates RNA polymerase III transcription in human cells. Mol. Cell Biol., 22: 3757-3768. DOI: 10.1128/MCB.22.11.3757-3768.2002

Lee, J.O., H. Yang, M.M. Georgescu, A. Di Cristofano and T. Maehama et al., 1999. Crystal structure of the PTEN tumor suppressor: Implications for its phosphoinositide phosphatase activity and membrane association. Cell, 99: 323-334. DOI: 10.1016/S0092-8674(00)81663-3

Leresche, A., V.J. Wolf and J.M. Gottesfeld, 1996. Repression of RNA polymerase II and III transcription during $\mathrm{M}$ phase of the cell cycle. Exp. Cell Res., 229: 282-288. DOI: 10.1006/excr.1996.0373

Leslie, N.R. and C.P. Downes, 2004. PTEN function: How normal cells control it and tumor cells lose it. Biochem. J., 382: 1-11. PMID: 15193142

Lockwood, W.W., R. Chari, B.P. Coe, K.L. Thu and C. Garnis et al., 2010. Integrative genomic analyses identify BRF2 as a novel lineage-specific oncogene in lung squamous cell carcinoma. PLoS Med., 7: e1000315-e1000315. DOI: 10.1371/journal.pmed.1000315

Marshall, L., N.S. Kenneth and R.J. White, 2008. Elevated $\mathrm{tRNA}_{\mathrm{i}}{ }^{\mathrm{Met}}$ synthesis can drive cell proliferation and oncogenic transformation. Cell, 133: 78-89. DOI: 10.1016/j.cell.2008.02.035

McMenamin, M.E., P. Soung, S. Perera, I. Kaplan and M. Loda et al., 1999. Loss of PTEN expression in paraffin-embedded primary prostate cancer correlates with high Gleason score and advanced stage. Cancer Res., 59: 4291-4296. PMID: 10485474 
Morton, J.P., T. Kantidakis and R.J. White, 2007. RNA polymerase III transcription is repressed in response to the tumor suppressor ARF. Nucleic Acids Res., 35: 3046-3052. DOI: 10.1093/nar/gkm208

Mossink, M.H., A. van Zon, R.J. Scheper, P. Sonneveld and E.A. Wiemer, 2003. Vaults: A ribonucleoprotein particle involved in drug resistance? Oncogene, 22: 7458-7467. PMID: 14576851

Odriozola, L., G. Singh, T. Hoang and A.M. Chan, 2007. Regulation of PTEN activity by its carboxylterminal autoinhibitory domain. J. Biol. Chem. 280: 35195-35202. DOI 10.1074/jbc.M61 1240200

Okumura, K., M. Zhao, R.A. DePinho, F.B. Furnari and W.K. Cavenee, 2005. PTEN: A novel antioncogenic function independent of phosphatase activity. Cell Cycle, 4: 540-542. PMID: 15753657

Planchon, S.M., K.A. Waite and C. Eng, 2008. The nuclear affairs of PTEN. J. Cell Sci., 121: 249-253. DOI: $10.1242 /$ jcs.022459

Raftopoulou, M., S. Etienne-Manneville, A. Self, S. Nicholls and A. Hall, 2003. Regulation of cell migration by the $\mathrm{C} 2$ domain of the tumor suppressor PTEN. Science, 303: 1179-1181. DOI: 10.1126/science.1092089

Ramaswamy, S., N. Nakamura, F. Vazquez, D.B. Batt and S. Perera et al., 1999. Regulation of G1 progression by the PTEN tumor suppressor protein is linked to inhibition of the phosphatidylinositol 3kinase/Akt pathway. Proc. Natl. Acad. Sci. USA., 96: 2110-2115. PMID: 10051603

Reina, J.H., T.N. Azzouz and N. Hernandez, 2006. Maf1, a new player in the regulation of human RNA polymerase III transcription. PLoS ONE, 1: e134- e134. DOI: 10.1371/journal.pone.0000134

Rollins, J., I. Veras, S. Cabarcas, I. Willis and L. Schramm, 2007. Human Maf1 negatively regulates RNA polymerase III transcription via the TFIIB family members Brf1 and Brf2. Int. J. Biol. Sci., 3: 292-302. PMID: 17505538

Schramm, L. and N. Hernandez, 2002. Recruitment of RNA polymerase III to its target promoters. Genes Dev., 16: 2593-2620. DOI: 10.1101/gad.1018902
Simpson, L. and R. Parsons, 2001. PTEN: Life as a tumor suppressor. Exp. Cell Res., 264: 29-41. DOI: 10.1006/excr.2000.5130

Sutcliffe, J.E., C.A. Cairns, A. McLees, S.J. Allison and K. Tosh et al., 1999. RNA polymerase III transcription factor IIIB is a target for repression by pocket proteins p107 and p130. Mol. Cell Biol., 19: 4255-4261. PMID: 10330166

Veras, I., E.M. Rosen and L. Schramm, 2009. Inhibition of RNA polymerase III transcription by BRCA1. J. Mol. Biol., 387: 523-531. DOI: 10.1016/j.jmb.2009.02.008

Waite, K.A. and C. Eng, 2002. Protean PTEN: Form and function. Am. J. Hum. Genet., 70: 829-844. DOI: $10.1086 / 340026$

Weng, L.P., W.M. Smith, P.L. Dahia, U. Ziebold and E. Gil et al., 1999. PTEN suppresses breast cancer cell growth by phosphatase activity-dependent G1 arrest followed by cell death. Cancer Res., 59: 5808-5814. PMID: 10582703

White, R.J., T.M. Gottlieb, C.S. Downes and S.P. Jackson, 1995. Cell cycle regulation of RNA polymerase III transcription. Mol. Cell Biol., 15: 6653-6662. PMID: 8524230

White, R.J., 2005. RNA polymerases I and III, growth control and cancer. Nat. Rev. Mol. Cell Biol., 6: 69-78. DOI: $10.1038 / \mathrm{nrm} 1551$

Woiwode, A., S.A.S. Johnson, S. Zhong, C. Zhang and R.G. Roeder et al., 2008. PTEN represses RNA polymerase III-dependent transcription by targeting the TFIIIB complex. Mol. Cell Biol., 28: 4204-4214. DOI: 10.1128/MCB.01912-07

Yu, Z., N. Fotouhi-Ardakani, L. Wu, M. Maoui and S. Wang et al., 2002. PTEN associates with the vault particles in HeLa cells. J. Biol. Chem., 277: 40247-40252. DOI: 10.1074/jbc.M207608200

Zhang, C., L. Comai and D.L. Johnson, 2005. PTEN represses RNA polymerase I transcription by disrupting the SL1 complex. Mol. Cell Biol., 25: 6899-6911. DOI: 10.1128/MCB.25.16.68996911.2005 